\title{
Spin-phonon coupling and pressure effect in the superconductor LiFeAs : Lattice dynamics from first-principles calculations
}

\author{
G. Q. Huang ${ }^{1}$, Z. W. Xing ${ }^{2}$, D. Y. Xing ${ }^{2}$ \\ ${ }^{1}$ Department of Physics and Institute of Theoretical Physics, \\ Nanjing Normal University, Nanjing 210046, China \\ ${ }^{2}$ National Laboratory of Solid State Microstructures, \\ Nanjing University, Nanjing 210093, China
}

\begin{abstract}
The lattice dynamics and the effect of pressure on superconducting LiFeAs in both nonmagnetic (NM) and striped antiferromagnetic (SAF) phases are investigated using the plane-wave pseudopotential, density-functional-based method. While the obtained electron-phonon coupling $\lambda$ is very small for the NM calculation, the softening of phonon in the SAF phase may lead to a large increase in $\lambda$. In the SAF phase, strong anisotropy of the phonon softening in the Fe plane is found to arise from different spin orders in the $x$ and $y$ directions, indicating that the phonon softening is of spin-phonon coupling origin. For the SAF structure, the calculated variation trend of the electronic density of states and the phonon frequencies under pressure can explain a large negative pressure coefficient of $T_{c}$ in the LiFeAs compound.
\end{abstract}

PACS numbers: $74.25 . \mathrm{Kc}, 74.70 . \mathrm{Xa}, 63.20 . \mathrm{dk}, 74.62 . \mathrm{Fj}$ 


\section{INTRODUCTION}

The discovery of superconductivity in layered iron pnictides $\frac{1}{1}$ has received tremendous attention. The superconducting transition temperature in doped $R e \mathrm{FeAsO}$ systems $(R e=\mathrm{Ce}$, Pr. Nd, Sm, ...) was quickly raised above $50 \mathrm{~K} .2 .3$ Other series including the doped $A \mathrm{Fe}_{2} \mathrm{As}_{2}$

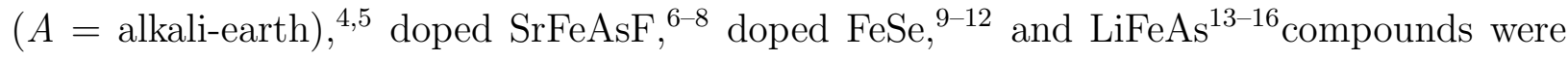
also found to be superconductors. All these compounds have formal FeAs layer, which consists of a square Fe lattice with As in the center of the square but being alternately shifted above and below the Fe plane. Both experimental and theoretical results showed that FeAs layer is the conducting layer, and dominates the main features near the Fermi level. $17-19$

The superconducting mechanism in iron pnictides is still under debate. Since the electronphonon (EP) interaction in the paramagnetic phase could only account for a maximum $T_{c}$ of $0.8 \mathrm{~K}, \frac{18}{\underline{n}}$ theoretical calculations ${ }^{18,20}$ ruled out phonon mediated superconductivity. On the other hand, McGuire et al..$^{21}$ suggested that strong electron-phonon coupling existed in the high temperature tetragonal phase of LaFeAsO, as evidenced by the behavior of the mobility, thermal conductivity, and Seebeck coefficient through the tetragonal-orthorhombic phasetransition region. The isotope effect ${ }^{22}$ was large in the pnictide superconductors, implying that the EP interaction should play an important role in the superconducting mechanism. Egamin et al ${ }^{23}$ suggested that the EP coupling through the spin channel might be sufficiently strong to be an important part of the superconductivity mechanism in Fe pnictides.

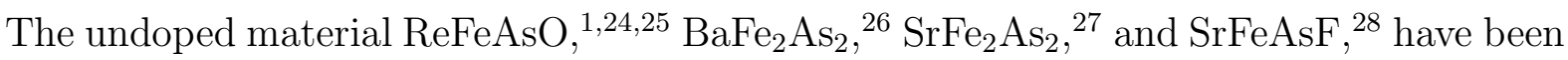
reported to undergo a spin-density wave transition. Upon doping, the spin-density wave is suppressed and superconductivity emerges $\stackrel{1,24-28}{2}$ First-principles calculations of LaFeAsO, 29 $\mathrm{BaFe}_{2} \mathrm{As}_{2}, \underline{30}$ and $\mathrm{SrFeAsF} \stackrel{31}{\underline{1}}$ all showed that the striped-antiferromagnetic (SAF) configuration is the stable ground state in these compounds. It was reported that, unlike the known other undoped intrinsic FeAs compounds, LiFeAs did not show any spin-density wave behavior but exhibits superconductivity at ambient pressures without chemical doping. $\underline{13}-\underline{16}$ But very recently, the experimental results provided direct evidence of the magnetic ordering in the nearly stoichiometric NaFeAs. $\frac{32}{2}$ The calculations for the nonmagnetic (NM) phase showed that the Fermi surface and band structures near Fermi level of LiFeAs are very similar to other iron pnictides. ${ }^{30,33}$ And the first-principles calculations $\frac{34,35}{3}$ showed 
that stoichiometric LiFeAs has almost the same SAF spin order as other FeAs-based parent compounds.

Many high pressure experiments on iron pnictides have been reported in the literature.

The superconductivity can be induced by pressure in undoped iron pnictides $\underline{36} \underline{37}$ For the doped iron pnictides, which are superconducting at ambient pressure, the pressure can

change their critical transition temperatures $\stackrel{25,38}{3}$ For LiFeAs, the experimental measurements showed that the superconducting $T_{c}$ decreases linearly with pressure at a rate of -1.5 $\mathrm{K} / \mathrm{GPa} \cdot \underline{\underline{39}} \underline{\underline{41}}$

In this paper we first calculate the electronic structure, phonon spectrum and the electronphonon interaction for the NM phase of LiFeAs compounds. The results obtained are consistent with those in previous works $\underline{30}, \underline{33}$ Since it is expected that the superconductivity is related to the spin order in the system, we investigate the lattice dynamics in the case of the SAF spin order. Our results show that the phonons from vibrations of the Fe and As atoms are softened due to the spin-lattice interaction, so as to enhance the electron-phonon coupling in the iron pnictides. The pressure effects for both NM and SAF phases are also investigated.

\section{COMPUTATIONAL METHOD}

The calculations have been performed in a plane-wave pseudo-potential representation through the PWSCF program. $\underline{\underline{42}}$ The ultrasoft pseudo-potential $\underline{\underline{43}}$ and general gradient approximation (GGA-PBE) $\underline{44}$ for the exchange and correlation energy functional are used with a cutoff of $30 \mathrm{Ry}$ for the wave functions and $240 \mathrm{Ry}$ for the charge densities. For the electronic structure calculations, the Brillouin zone integrations are performed by using the Gaussian smearing technique with a width of 0.04 Ry. Within the framework of the linear response theory, the dynamical matrixes and the electron-phonon interaction coefficients are calculated. The energy and frequency convergence are checked with respect to the cutoff energy and k-point sampling. 


\section{RESULTS AND DISCUSSION}

\section{A. Calculation in the NM phase}

We first do the first-principles calculations for LiFeAs in the NM case, and then compare our results with the previous works $\underline{\underline{30}, \underline{33}}$ The LiFeAs compound crystallizes in a PbFCl-type structure (space group P4/nmm). The Wyckoff positions for Fe, Li, and As are $2 b, 2 c$, and $2 c$, respectively. Structural optimizations involving the lattice constants and the internal coordinates are performed using the Broyden-Fletcher-Goldfarb-Shanno algorithm. $\underline{\underline{45}}$ The obtained lattice constants, $(a, c)=(3.83,6.55) \AA$, are slightly larger than the experimental values $(3.79,6.36) \AA \stackrel{13}{\underline{13}}$ The obtained internal coordinate, $z_{L i}=0.3464$, is close to the experimental value of $0.3459, \frac{13}{\underline{1}}$ but the obtained $z_{A s}=0.2083$ is noticeably lower than the reported value of $z_{A s}=0.2365 \underline{\underline{13}}$ The resulting height difference is about $0.15 \AA$.

The electronic structure calculation agrees well with previous calculations for the nonspin-polarized situation. LiFeAs belongs to be the low carrier density metal with high density of states. The calculated density of states at the Fermi level, $N\left(E_{F}\right)$, is equal to $3.71 \mathrm{eV}^{-1}$ per unit cell (two formula units), which is in between $3.86 \mathrm{eV}^{-1}$ obtained in Ref. 33 and $3.58 \mathrm{eV}^{-1}$ in Ref. 30 . The states near the Fermi level are dominated by Fe $3 d$ states lightly mixed with As $p$ states. The Fermi surface consists of hole cylinders centered at the zone center and electron cylinders centered at the zone corner. In general, the electronic structure near the Fermi level of LiFeAs is qualitative similar to that of the other FeAs materials.

The calculated phonon dispersion of LiFeAs along major high symmetry directions of the Brillouin zone is plotted in Fig. 1. The vibrations of Fe and As atoms occupy the whole energy range, while the vibrations of Li atoms occupy the high-frequency region because of its very small mass. We notice that the Fe-As bond-stretching modes are on the side of high frequency. In fact, the modes having the same symmetry may be coupled with each other. The phonon eigenvectors have a strongly mixed character and cannot be simply traced back to a single vibration pattern. In the high-frequency region, our calculated phonon spectrum is different from that obtained previously by Jishi et al.. $\underline{46}$ The top bands for the vibrations of Li atoms are separated from other bands in Ref. 46, while they are coupled with other bands in our calculations. This difference may stem from different lattice constants and atomic internal coordinates used in calculations. In this paper, we use the optimized 
lattice constants and atomic internal coordinates, while Jishi et al. used the corresponding experimental values. The frequencies involving the vibrations of Li atoms are sensitive to computational details due to very small atomic mass.

The EP coupling constant $\lambda$ and the logarithmically averaged frequency $\omega_{l n}$ are directly obtained by evaluating

$$
\lambda=2 \int_{0}^{\infty} \frac{\alpha^{2} F(\omega)}{\omega} d \omega
$$

and

$$
\omega_{l n}=\exp \left(\frac{2}{\lambda} \int_{0}^{\infty} \frac{\alpha^{2} F(\omega) \ln \omega}{\omega} d \omega\right),
$$

where the EP spectral function $\alpha^{2} F(\omega)$ can be determined self-consistently by the linear response theory. The calculated results are $\lambda=0.26$ and $\omega_{l n}=236 \mathrm{~K}$. Using the McMillan expression for $T_{c}$ and taking the Coulomb pseudopotential parameter $\mu^{*}=0.1$, the resulting value of $T_{c}$ is much less than $1 \mathrm{~K}$. This is consistent with the result reported by Jishi et al.. $\underline{46} \mathrm{It}$ then followed that the electron-phonon coupling is too weak to account for superconductivity in LiFeAs. However, the phonon mediated superconductivity cannot be ruled out only according to the conclusion in the NM case.

\section{B. Spin-lattice interaction}

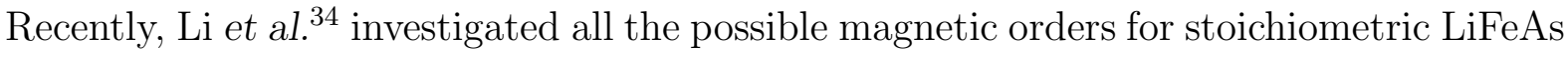
by using the full-potential linearized augmented plane wave method, and concluded that the magnetic ground state of LiFeAs is an SAF order in each Fe layer and a weak antiferromagnetic order in the $z$ direction. In this paper we have done the same calculations by using the plane-wave pseudo-potential method. Our results confirm the conclusion in Ref. 34 that stoichiometric LiFeAs has almost the same SAF spin order as other FeAs-based parent compounds. Next, we investigate the lattice dynamics for this SAF spin order. Since the antiferromagnetic interaction in the $z$ direction is very weak, $\underline{\underline{34}}$ we will not consider it in the following calculations.

To accommodate the SAF magnetic structure, we use a $\sqrt{2} \times \sqrt{2} \times 1$ supercell. The magnetic structure in the Fe layer is that the Fe spins align ferromagnetically in the $x$ direction and antiferromagnetically in the $y$ direction. We have performed the full structural optimization, including the shape and size of the unit cell as well as the atomic internal positions. 
The result is that the lattice structure is distorted from the tetragonal (higher) symmetry to orthogonal (lower) symmetry with lattice constants as $(a, b, c)=(5.462,5.559,6.776) \stackrel{\circ}{A}$. They are larger than the experimental values, especially along the $z$ direction; whereas in Refs. [34] and [35] the calculated equilibrium volume of the SAF magnetic structure by the projector augmented wave method is somewhat smaller than the experimental values. The obtained internal parameters of $\mathrm{Li}$ and As atoms are 0.3504 and 0.2206, respectively. Our calculated heights of the As atoms from the Fe plane for the NM and SAF structures are $1.364 \AA$ and $1.495 \AA$, respectively. The corresponding experimental value is $1.504 \AA$, 13 which is closer to the calculated value in the presence of the SAF ordering. This conclusion is consistent with those in previous references $34, \underline{35,47}-\underline{49}$. The obtained magnetic moment per Fe atom by the GGA calculation in this paper is about $2.60 \mu \mathrm{B}$. According to other studies 34,35 , the magnetic moment calculated by the local spin-density approximation is much smaller than that by the GGA calculation. Next, we calculate all zone-center phonon frequencies for the SAF magnetic structure within the framework of the linear response theory. For comparison, we also calculate the zone-center phonon frequencies for the NM structure with the same $\sqrt{2} \times \sqrt{2} \times 1$ supercell. However, it is difficult to directly compare the changes of phonon frequencies because of different irreducible representations in the two structures.

To investigate how the spin order in SAF magnetic structure changes the phonon frequency in LiFeAs, we define a weighted average frequency as

$$
\bar{\omega}_{\alpha, n}=\sum_{\nu} \frac{u_{\alpha, n, \nu}^{2}}{\left|\mathbf{u}_{\nu}\right|^{\mathbf{2}}} \omega_{\nu}
$$

where $\alpha$ is the polarization index $(x, y, z), n$ is the number of atom index, and the sum runs over all the modes. $\left|\mathbf{u}_{\nu}\right|$ is the amplitude of the atomic displacements for the $\nu$ th normal mode and $u_{\alpha, n, \nu}^{2} /\left|\mathbf{u}_{\nu}\right|^{2}$ represents the percentage of the contribution from the $\alpha$ polarization direction of the $n$th atom. The calculated average frequencies for the NM and SAF structures at the theoretical zero pressure $(\mathrm{P}=0)$ are listed in Table 1 , in which the relative changes of frequency $\left(\frac{\bar{\omega}_{S A F}-\bar{\omega}_{N M}}{\bar{\omega}_{N M}} \times 100 \%\right)$ are also given. An evident feature in Table 1 is the softening of the phonon for Fe and As atoms in the SAF magnetic structure relative to that in the NM structure. Furthermore, the softening of phonons is nearly isotropic in the in-plane directions for the As atoms, but strongly anisotropic for the Fe atom. The most softening appears for $\bar{\omega}_{x}$ of the Fe atom. It changes from $216.92 \mathrm{~cm}^{-1}$ in the NM structure to 148.68 $\mathrm{cm}^{-1}$ in the SAF magnetic structure, reducing about $31 \%$. By comparison, $\bar{\omega}_{y}$ of the Fe 
atom reduces only about $14 \%$.

To further investigate behavior of the phonon softening, we have examined the As $A_{g}$ mode and Fe $E_{g}$ mode (named as in the tetragonal structure) with the frozen-phonon calculation. Both the two modes are coupled with other modes according to the symmetry. In the frozen-phonon calculation, we don't consider the coupling with vibrations of other atoms. For the As $A_{g}$ mode, As atoms above and below the Fe plane displace along $z$ in opposite directions. The calculated distorted energies versus atom displacements are plotted in Fig. 2a, which can be well fitted to $E=k_{2} u^{2}$ of harmonic modes. It can be clearly seen that the energy curve, $E=27.70 u^{2}$, obtained in the NM structure is steeper than $E=13.87 u^{2}$ in the SAF structure. The calculated frequencies $\omega$ are $28.29 \mathrm{meV}$ and $22.62 \mathrm{meV}$ for the NM structure and SAF structures, respectively, yielding a reducing of about $20 \%$. Since the Fe-magnetic moment is sensitive to the As-z position, the calculated Fe-magnetic moment versus the As displacement is also plotted in the insert of Fig. 2a. With increasing the distance between the As atom and the Fe plane, the obtained Fe-magnetic moment increases, which has the same trend in LaOFeAs as calculated by Yildirim ${ }^{50}$.

For the in-plane Fe $E_{g}$ mode, from the higher tetragonal symmetry to lower orthogonal symmetry, the doubly degenerate mode will split into non-degenerate ones, as shown in the insert of Fig. 2b. For the doubly degenerate mode in the NM structure, the distorted energy can be well fitted to quadratic equation $E=30.04 u^{2}$, and the obtained frequency is $33.29 \mathrm{meV}$. For the two non-degenerate modes in the SAF structure, the distorted energy may be fitted to $E=k_{2} u^{2}+k_{4} u^{4}$, where $k_{4} u^{4}$ is the anharmonic term of the mode. Using the Hartree-Fock decoupling, one gets $E(u)=\left(k_{2}+3 k_{4}\left\langle u^{2}\right\rangle\right) u^{2}$, where $\left\langle u^{2}\right\rangle=\hbar / 2 M \omega_{\text {sch }}$ and $\omega_{\text {sch }}=\left[\left(k_{2}+3 k_{4}\left\langle u^{2}\right\rangle\right) / M\right]^{1 / 2}$ is the self-consistent phonon frequency. ${ }^{51}$ For the Fe atom vibration along the $x(y)$ direction, we obtain $E=13.70 u^{2}+59.52 u^{4}\left(E=21.71 u^{2}+25.24 u^{4}\right)$, the harmonic frequency $\omega=22.48 \mathrm{meV}(28.30 \mathrm{meV})$, and the self-consistent phonon frequency $\omega_{\text {sch }}=22.80 \mathrm{meV}(28.47 \mathrm{meV})$. The anharmonic effect leads to a slight enhancement in the phonon frequency. From the NM structure to the SAF structure, the phonon frequency decreases about $31.5 \%(14.5 \%)$ for the Fe atom vibration along the $x(y)$ direction. It is interesting to note that the present softening of phonon frequencies obtained by the frozenphonon calculation is quite well consistent with that of $\bar{\omega}_{x}$ and $\bar{\omega}_{y}$ obtained above by the linear response perturbation theory.

The conclusion of the phonon softening in the SAF magnetic phase agrees well with the 
results studied by others. The first-principles calculations by Fukuda et al. $\underline{\underline{52}}$ for the NM structure in $\mathrm{LaFeAsO}_{1-x} \mathrm{~F}_{x}$ and $\mathrm{PrFeAsO}_{1-y}$ indicated that the calculated phonon DOS could agree with the experimental spectrum only when the computed Fe-As force constant is reduced by $30 \%$. The calculations by Yildirim ${ }^{50}$ showed that the in-plane Fe-Fe and $c$ polarized As phonon modes are softened about $10 \%$ for the LaOFeAs system, and softened about $10-14 \%$ and $23 \%$ for the $\mathrm{BaFe}_{2} \mathrm{As}_{2}$, explaining the experimental data ${ }^{53,54}$. As a result, it can be concluded that the SAF magnetic structure has significant effects on the phonon frequency, which may be a common feature in iron pnictides.

The difference of lattice constants $a$ and $b$ in the orthogonal structure is very small $(b / a=1.02)$, but the difference of the phonon softening along $x$ and $y$ direction for $\mathrm{Fe}$ atoms is large. This seems to indicate that the difference in phonon softening arises from the anisotropic spin order rather than the change of the geometric structure. In order to confirm this point, we have carried out calculations by the linear response perturbation theory for both NM and magnetic SAF phases, in which the lattice parameters are fixed to the experimental values of the tetragonal structure and other internal parameters are optimized. The calculated results for the ambient pressure are listed in the top rows of Table 1. It is found that there is large phonon softening for the vibrations of both Fe and As atoms; and that for the Fe atoms, the magnitude of softening in the $x$ direction is larger than that in the $y$ direction. It then follows that the spin-lattice interaction is the origin of the softening. It is the different spin orders in the $x$ and $y$ directions that result in strong anisotropy of the phonon softening in the Fe plane.

Owing to the presence of strong spin-lattice interactions, it is imperative to study the phonon-mediated mechanism via the spin channel. Yildirim ${ }^{29}$ suggested that magnetism and superconductivity in doped LaFeAsO may be strongly coupled, much like in the highTc cuprates. The large iron isotope effect found by Liu et al $\underline{\underline{22}}$ for both spin-density wave and superconducting transition temperatures seemed to indicate that phonons not only play a dominant role for superconductivity, but also are close coupled with magnetism in the iron pnictides. Egami et al $\stackrel{23}{\underline{2}}$ suggested that the EP coupling through the spin channel may be sufficiently strong to be an important part of the superconductivity mechanism in Fe pnictides. According to Eq. (1), we can see that the softening of phonons in the SAF phases is favorable to increasing the EP coupling constant $\lambda$, and an increasing $\lambda$ will lead to an enhancement of $T_{c}$. 


\section{Pressure effect}

For LiFeAs, the experimental measurements showed that the superconducting $T_{c}$ decreases linearly with pressure at a rate of $-1.5 \mathrm{~K} / \mathrm{GPa} \cdot \stackrel{39-41}{2}$ Here we investigate the pressure effect for both NM and SAF phases. Constant pressure molecular dynamics is performed to optimize the cell size, volume, and atomic internal positions. The optimized parameters together with some calculated results are listed in Table 2 . The lattice constants, the volume of the unit supercell, and the Fe-As bond length decrease monotonically with increasing the pressure up to $15 \mathrm{GPa}$. The decrease of $c / a$ under pressure indicates that the out-of-plane compression is larger than the in-plane ones. From Table 2, we can see that the decrease of percentage of the volume in the SAF phase is larger than that in the NM phase under the same pressure, indicating that the compression in the SAF phase is easier than that in the NM phase. For example, from the theoretical zero pressure to $1.5 \mathrm{Gpa}$, the volume decreases $4.88 \%$ in the SAF structure while it decreases only $3.31 \%$ in the NM structure. We also calculate the bulk modulus $B$ by fitting the $E$ (total energy) versus $V$ (volume) curves to the Birch-Murnagham equation of state. The obtained $B$ are 454 and $353 \mathrm{kbar}$ for the NM and SAF, respectively. The smaller bulk modulus $B$ in the SAF structure means weaker interactions between atoms and smaller force constants, resulting in a lower frequency of phonon. This is just consistent with the calculated results discussed above.

The density of states at the Fermi level, $N\left(E_{F}\right)$, is also listed in Table 2. In the range up to $15 \mathrm{Gpa}, N\left(E_{F}\right)$ decreases with compression in the SAF structure, as expected as usual. In general, a compression of the lattice by pressure causes an increase of the bandwidth, which in turn results in a decrease of the averaged density of states. For the NM phase, the Fermi level lies just in the flat region of the DOS curve, and so $N\left(E_{F}\right)$ changes little under pressure. For the SAF structure, the calculated magnetic moment each iron atom decreases as the pressure is increased, which has the same trend as the calculation by Nakamura et al. $\stackrel{54}{*}$ for the LaFeAsO compound.

Next, we wish to study the pressure effect on the phonon frequency. The calculated average frequencies at $\mathrm{p}=1.5 \mathrm{Gpa}$ and $15 \mathrm{Gpa}$ for the two structures are also listed in Table 1. As usual, all the calculated frequencies increase with pressure, for a smaller lattice constant leads to a stronger force constant. For the calculated average frequencies of the Fe and As atoms, however, the increase in the SAF structure is much larger than that in 
the NM structure. From Table 1, one also finds that Up to 15Gpa, the phonon softening from the NM to SAF phase and its anisotropy in the Fe plane still exist, but such a phonon softening decreases with the increase of pressure. Relative to those in the NM structure, the calculated average frequencies of the Fe atom in the SAF structure reduce about $31.5 \%$ and $13.8 \%$ for $\bar{\omega}_{x}$ and $\bar{\omega}_{y}$, respectively, at the theoretical zero pressure. Correspondingly, they reduce only about $12.5 \%$ and $6.4 \%$ for $\bar{\omega}_{x}$ and $\bar{\omega}_{y}$, respectively, at $15 \mathrm{Gpa}$. It then follows that the difference in some physical properties between the NM and SAF structures becomes small with increasing pressure. The SAF structure may not be the ground structure when the pressure is increased to some extent, as has been found in $\mathrm{LaOFeAs}^{54}$.

For the SAF structure, our calculations show that with increasing pressure, $N\left(E_{F}\right)$ decreases and the phonon frequencies increase greatly, resulting in a large decrease of the EP coupling constant $\lambda$. All these factors will lead to a lower $T_{c}$ and a large negative pressure coefficient of $T_{c}$. As a result, the pressure effect on superconducting $T_{C}$ is not contradictory to the EP coupling mechanism provided that the spin degree of freedom is taken into account.

\section{Summary}

The electronic structure, phonon spectrum and the EP interaction in the NM LiFeAs compound have been investigated by the first-principles calculations. The obtained electronphonon coupling for the NM calculation is too weak to account for superconductivity in LiFeAs. After considering the spin order, the calculated average frequencies, especially from the vibrations of Fe and As atoms in the SAF structure, are smaller than those in the NM structure. It is found that the different spin orders in the $x$ and $y$ directions result in strong anisotropy of the softening of phonons in the Fe plane. It then follows that the origin of the phonon softening is the spin-lattice interaction. The softening of phonons in the SAF phase can lead to an increase of EP coupling constant $\lambda$ and superconducting temperature $T_{c}$. By the calculations under different pressures, the obtained bulk modulus $B$ in the SAF structure is smaller than that in the NM structure, indicating that the compression in the SAF phase is easier than that in the NM phase. For the SAF structure, our calculations show that with increasing pressure, both the decrease of the density of states at the Fermi

level and the increase of the phonon frequencies make $\lambda$ and $T_{c}$ decrease, resulting in a large 
negative pressure coefficient of $T_{c}$.

\section{Acknowledgments}

G.Q.H. and D.Y.X. acknowledge support from the National Natural Science Foundation of China under Grant No. 10947005, Z.W.X acknowledges support from the Natural Science Foundation of Jiangsu Province in China under Grant No. SBK200920627, and G.Q.H. acknowledges support from the Foundation of Jiangsu Education Office of China under Grant No. 09KJB140004. This work is also supported by the State Key Program for Basic Researches of China under Grants No. 2006CB921803 and No. 2010CB923400.

1 Y. Kamihara, T. Watanabe, M. Hirano, and H. Hosono, J. Am. Chem. Soc. 130, 3296 (2008).

2 Z.-A Ren, J. Yang, W. Lu, W. Yi, X.-L Shen, Z.-G Li, G.-C Che, X.-L Dong, L.-L Sun, F. Zhou, Z.-X Zhao, Eur. Phys. Lett. 82, 57002 (2008).

3 X. H. Chen, T. Wu, G. Wu, R. H. Liu, H. Chen, D. F. Fang, Nature 453, 761 (2008).

4 M. Rotter, M. Tegel, and D. Johrendt,Phys. Rev. Lett. 101, 107006 (2008).

5 K. Sasmal, B. Lv, B. Lorenz, A.M. Guloy, F. Chen, Y.Y. Xue, and C. W. Chu, Phys. Rev. Lett. 101, 107007 (2008).

6 S. Matsuishi, Y. Inoue, T. Nomura, M. Hirano, and H. Hosono, J. Phys. Soc. Jpn. 77, 113709 (2008).

7 G. Wu, Y. L. Xie, H. Chen, M. Zhong, R. H. Liu, B. C. Shi, Q. J. Li, X. F. Wang, T. Wu, Y. J. Yan, J. J. Ying, X. H. Chen,Journal of Physics: Condensed Matter 21, 142203(2009).

8 Xiyu Zhu, Fei Han, Peng Cheng, Gang Mu, Bing Shen, Hai-Hu Wen, Europhys. Lett. 85, 17011 (2009).

9 Y. Mizuguchi, F. Tomioka, S. Tsuda, T. Yamaguchi and Y. Takano, Appl. Phys. Lett. 93, 152505 (2008).

10 A. Subedi, L. Zhang, D. J. Singh, and M. H. Du, Phys. Rev. B 78, 134514 (2008).

11 Y. F. Li, L. F. Zhu, S. D. Guo, Y. C. Xu, and B. G. Liu, J. Phys: Condens. Matter 21, 115701 (2009). 
12 F. C. Hsu, J. Y. Luo, K. W. Yeh, T. K. Chen, T. W. Huang, P. M. Wu, Y. C. Lee, Y. L. Huang, Y. Y. Chu, D. C. Yan, and M. K Wu, Proc. Natl. Acad. Sci. USA 105, 14262 (2008).

13 J. H. Tapp, Z. Tang, B. Lv, K. Sasmal, B. Lorenz, P. C. W. Chu, and A. M. Guloy, Phys. Rev. B 78, 060505(R) (2008).

14 M. J. Pitcher, D. R. Parker, P. Adamson, S. J. C. Herklrath, A. T. Boothroyd, R. M. Ibberson, M. Brunelli, and S. J. Clarke, Chem. Commun. 2008, 5918 (2008).

15 X. C. Wang, Q. Q. Liu, Y. X. Lv, W. B. Gao, L. X. Yang, R. C. Yu, F.Y. Li, and C. Q. Jin, Solid State Commun. 148, 538 (2008).

16 C.W. Chu, F. Chen, M. Gooch, A. M. Guloy, B. Lorenz, B. Lv, K. Sasmal, Z. J. Tang, J. H. Tapp, and Y.Y. Xue, arXiv:0902.0806.

17 H. W. Ou, J. F. Zhao, Y. Zhang, D. W. Shen, B. Zhou, L. X. Yang, C. He, F. Chen, M. Xu, T. Wu, X. H. Chen, Y. Chen, D. L. Feng,Chin. Phys. Lett. 25, 2225(2008).

18 L. Boeri, O.V. Dolgov, and A. A. Golubov, Phys. Rev. Lett. 101, 026403 (2008).

19 D. J. Singh and M.-H. Du, Phys. Rev. Lett. 100, 237003 (2008).

20 K. Haule, J. H. Shim, and G. Kotliar, Phys. Rev. Lett. 100, 226402 (2008).

21 M. A. McGuire, A. D. Christianson, A. S. Sefat, B. C. Sales, M. D. Lumsden, R. Jin, E. A. Payzant, D. Mandrus, Y. Luan, V. Keppens, V. Varadarajan, J. W. Brill, R. P. Hermann, M. T. Sougrati, F. Grandjean, and G. J. Long, Phys. Rev. B 78, 094517 (2008).

22 R. H. Liu, T. Wu, G. Wu, H. Chen, X. F. Wang, Y. L. Xie, J. J. Yin, Y. J. Yan, Q. J. Li, B. C. Shi, W. S. Chu, Z. Y. Wu, X. H. Chen, Nature 459, 64-67(2009).

23 T. Egami, B. V. Fine, D. Parshall, A. Subedi and D. J. Singh, Advances in Condensed Matter Physics, 2010164916 (2010).

24 J. Dong, H. J. Zhang, G. Xu, Z. Li, G. Li, W. Z. Hu, D. Wu, G. F. Chen, X. Dai, J. L. Luo, Z. Fang, and N. L. Wang, Europhys. Lett. 83, 27006 (2008).

25 B. Lorenz, K. Sasmal, R. P. Chaudhury, X. H. Chen, R. H. Liu, T. Wu, and C. W. Chu, Phys. Rev. B 78, 012505 (2008).

26 Q. Huang, Y. Qiu, Wei Bao, M. A. Green, J. W. Lynn, Y. C. Gasparovic, T. Wu, G. Wu, and X. H. Chen, Phys. Rev. Lett. 101, 257003 (2008).

27 G. F. Chen, Z. Li, J. Dong, G. Li, W. Z. Hu, X. D. Zhang, X. H. Song, P. Zheng, N. L. Wang, J. L. Luo, Phys. Rev. B 78, 224512 (2008).

28 Fei Han, Xiyu Zhu, Gang Mu, Peng Cheng, Hai-Hu Wen, Phys. Rev. B 78, 180503(R) (2008). 
29 T. Yildirim, Phys. Rev. Lett. 101, 057010 (2008).

30 D. J. Singh, Phys. Rev. B 78, 094511 (2008).

31 L. F. Zhu, B. G. Liu, EuroPhys. Lett. 85, 67009 (2009).

32 G. F. Chen, W. Z. Hu, J. L. Luo, and N. L. Wang, Phys. Rev. Lett. 102, 227004 (2009).

33 I.A. Nekrasov, Z.V. Pchelkina, M.V. Sadovskii,JETP Letters 88, 543 (2008).

34 Yong-Feng Li, Bang-Gui Liu ,Eur. Phys. J. B 72, 153 (2009).

35 Zhi Li, J. S. Tse, and C. Q. Jin, Phys. Rev. B 80, 092503 (2009).

36 M. S. Torikachvili, S. L. Budko, N. Ni, and P. C. Canfield, Phys. Rev. Lett. 101, 057006 (2008).

37 C. F. Miclea, M. Nicklas, H. S. Jeevan, D. Kasinathan, Z. Hossain, H. Rosner, P. Gegenwart, C. Geibel, and F. Steglich, Phys. Rev. B 79, 212509 (2009).

38 H. Takahashi, K. Igawa, K. Arii, Y. Kamihara, M. Hirano, and H. Hosono, Nature 453, 376 (2008).

39 M. Gooch, B. Lv, J. H. Tapp, Z. Tang, B. Lorenz, A. M. Guloy, P. C. W. Chu, EuroPhys. Lett. 85, 27005 (2009).

40 S. J. Zhang, X. C. Wang, R. Sammynaiken, J. S. Tse, L. X. Yang, Z. Li, Q. Q. Liu, S. Desgreniers, Y. Yao, H. Z. Liu, and C. Q. Jin, Phys. Rev. B 80, 014506 (2009)

41 C. W. Chu, B. Lorenz, arXiv:0902.0809v1 [cond-mat.supr-con]

42 S. Baroni, S. de Gironcoli, A. Dal Corso and P. Giannozzi, Available from: http://www.pwscf.org

43 D. Vanderbilt, Phys. Rev. B 41, 7892 (1990).

44 J.P. Perdew, K. Burke and M. Ernzerhof, Phys. Rev. Lett. 77, 3865 (1996).

45 S. R. Billeter, A Curioni and W. Andreoni, Comput. Mater. Sci. 27, 437 (2003).

46 R. A. Jishi, H. M. Alyahyaei, Advances in Condensed Matter Physics, vol. 2010, Article ID 804343, 6 pages, 2010

47 T. Yildirim, Phys. Rev. Lett. 102, 037003 (2009).

48 Z. P. Yin, S. Lebegue, M. J. Han, B. P. Neal, S. Y. Savrasov, and W. E. Pickett, Phys. Rev. Lett. 101, 047001 (2008).

49 I. I. Mazin and M. D. Johannes, Nature Physics 5, 141 (2009).

50 T. Yildirim, Physica C 469, 425 (2009).

51 Phonons in Perfect Lattices and in Lattices with Point Imperfections, edited by R.W. H. Stevenson (Plenum Press, New York, 1966). 
52 T. Fukuda, A. Q. R. Baron, S. Shamoto, M. Ishikado, H. Nakamura, M. Machida, H. Uchiyama, S. Tsutsui, A. Iyo, H. Kita, J. Mizuki, M. Arai, H. Eisaki, Y. Matsuda, and H. Hosono, J. Phys. Soc. Jpn. 77, 103715 (2008).

53 D. Reznik, K. Lokshin, D. C. Mitchell, D. Parshall, W. Dmowski, D. Lamago, R. Heid, K.-P. Bohnen, A. S. Sefat, M. A. McGuire, B. C. Sales, D. G. Mandrus, A. Subedi, D. J. Singh, A. Alatas, M. H. Upton, A. H. Said, A. Cunsolo, Yu. Shvyd'ko, and T. Egami, Phys. Rev. B 80, $214534(2009)$.

54 Hiroki Nakamura and Masahiko Machida, Phys. Rev. B 80, 165111 (2009). 
TABLE I: The calculated average frequency $\left(\mathrm{cm}^{-1}\right)$ at different pressures. For the ambient pressure, the lattice parameters are fixed to the experimental values of the tetragonal structure and the internal parameters are optimized.

\begin{tabular}{|c|c|c|c|}
\hline & $\left(\bar{\omega}_{x}, \bar{\omega}_{y}, \bar{\omega}_{z}\right)$ for $\mathrm{Fe}$ & $\left(\bar{\omega}_{x}, \bar{\omega}_{y}, \bar{\omega}_{z}\right)$ for As & $\left(\bar{\omega}_{x}, \bar{\omega}_{y}, \bar{\omega}_{z}\right)$ for $\mathrm{Li}$ \\
\hline \multicolumn{4}{|l|}{ ambient pressure } \\
\hline NM phase & $(224.69,224.69,207.50)$ & $(173.70,173.70,183.27)$ & $(233.02,233.02,299.80)$ \\
\hline SAF phase & $(175.44,212.30,192.59)$ & $(152.15,149.95,164.09)$ & $(232.65,245.07,330.25)$ \\
\hline$\frac{\bar{\omega}_{S A F}-\bar{\omega}_{N M}}{\bar{\omega}_{N M}} \times 100(\%)$ & $(-21.92,-5.51,-7.19)$ & $(-12.41,-13.67,-10.47)$ & $(-0.16,5.17,10.16)$ \\
\hline \multicolumn{4}{|l|}{$\mathrm{P}=0(\mathrm{Gpa})$} \\
\hline NM phase & $(216.92,216.92,198.59)$ & $(170.36,170.36,174.69)$ & $(210.01,210.01,249.66)$ \\
\hline SAF phase & $(148.68,187.03,170.22)$ & $(141.73,139.17,142.20)$ & $(194.88,191.49,221.98)$ \\
\hline$\frac{\bar{\omega}_{S A F}-\bar{\omega}_{N M}}{\bar{\omega}_{N M}} \times 100(\%)$ & $(-31.46,-13.78,-14.29)$ & $(-16.81,-18.31,-18.60)$ & $(-7.20,-8.82,-11.09)$ \\
\hline \multicolumn{4}{|l|}{$\mathrm{P}=1.5(\mathrm{Gpa})$} \\
\hline NM phase & $(220.47,220.47,203.67)$ & $(172.64,172.64,180.29)$ & $(225.82225 .82,282.33)$ \\
\hline SAF phase & $(158.20,192.67,176.43)$ & $(145.51,142.48,151.46)$ & $(213.77,205.11,267.77)$ \\
\hline$\frac{\bar{\omega}_{S A F}-\bar{\omega}_{N M}}{\bar{\omega}_{N M}} \times 100(\%)$ & $(-28.24,-12.61,-13.37)$ & $(-15.71,-17.47,-15.99)$ & $(-5.34,-9.17,-5.16)$ \\
\hline \multicolumn{4}{|l|}{$\mathrm{P}=15(\mathrm{Gpa})$} \\
\hline NM phase & $(249.15,249.15,232.44)$ & $(186.26,186.26,208.41)$ & $(312.79,312.79,427.45)$ \\
\hline SAF phase & $(218.02,233.29,218.76)$ & $(166.53,160.47,186.53)$ & $(328.32,300.24,413.71)$ \\
\hline$\frac{\bar{\omega}_{S A F}-\bar{\omega}_{N M}}{\bar{\omega}_{N M}} \times 100(\%)$ & $(-12.49,-6.37,-5.89)$ & $(-10.59,-13.85,-10.50)$ & $(4.96,-4.01,-3.21)$ \\
\hline
\end{tabular}


TABLE II: Crystal structure data and the density of states at the Fermi level $N\left(E_{F}\right)$ with $\sqrt{2} \times$ $\sqrt{2} \times 1$ supercell under different pressures. $V_{0}$ means the theoretical equilibrium volume

\begin{tabular}{|c|c|c|c|c|c|c|}
\hline $\mathrm{p}(\mathrm{Gpa})$ & $(\operatorname{Exp})^{a}$ & 0 & 0.5 & 1.0 & 1.5 & 15 \\
\hline \multicolumn{7}{|l|}{ NM phase } \\
\hline $\mathrm{a}(\stackrel{\circ}{A})$ & 5.3610 & 5.4168 & 5.4078 & 5.3950 & 5.3795 & 5.1645 \\
\hline $\mathrm{c} / \mathrm{a}$ & 1.1872 & 1.2094 & 1.2042 & 1.2011 & 1.1939 & 1.1578 \\
\hline $\mathrm{V}\left(\stackrel{\circ}{A}^{3}\right)$ & 182.920 & 192.220 & 190.441 & 188.605 & 185.863 & 159.484 \\
\hline$\left(\mathrm{V}-\mathrm{V}_{0}\right) / \mathrm{V}_{0} \times 100(\%)$ & & 0 & -0.93 & -1.88 & -3.31 & -17.03 \\
\hline $\mathrm{z}(\mathrm{As})$ & 0.2365 & 0.2083 & 0.2096 & 0.2110 & 0.2136 & 0.2355 \\
\hline $\mathrm{z}(\mathrm{Li})$ & 0.3459 & 0.3465 & 0.3466 & 0.3469 & 0.3481 & 0.3445 \\
\hline$d_{F e-A s}(\stackrel{\AA}{A})$ & 2.4204 & 2.3516 & 2.3491 & 2.3467 & 2.3451 & 2.3058 \\
\hline$N\left(E_{F}\right)($ States $/ \mathrm{eV})$ & & 7.424 & 7.372 & 7.346 & 7.350 & 7.456 \\
\hline \multicolumn{7}{|l|}{ SAF phase } \\
\hline $\mathrm{a}(\stackrel{\circ}{A})$ & & 5.4616 & 5.4194 & 5.4039 & 5.3890 & 5.0712 \\
\hline $\mathrm{b} / \mathrm{a}$ & & 1.0178 & 1.0183 & 1.0186 & 1.0187 & 1.0205 \\
\hline $\mathrm{c} / \mathrm{a}$ & & 1.2405 & 1.2357 & 1.2340 & 1.2273 & 1.2142 \\
\hline $\mathrm{V}\left(\stackrel{\circ}{A}^{3}\right)$ & & 205.693 & 200.282 & 198.353 & 195.669 & 161.598 \\
\hline$\left(\mathrm{V}-\mathrm{V}_{0}\right) / \mathrm{V}_{0} \times 100(\%)$ & & 0 & -2.631 & -3.568 & -4.873 & -21.437 \\
\hline $\mathrm{z}(\mathrm{As})$ & & 0.2206 & 0.2242 & 0.2247 & 0.2263 & 0.2439 \\
\hline $\mathrm{z}(\mathrm{Li})$ & & 0.3504 & 0.3532 & 0.3541 & 0.3550 & 0.3496 \\
\hline$d_{F e-A s}(\AA)$ & & 2.4554 & 2.4481 & 2.4421 & 2.4369 & 2.3530 \\
\hline$N\left(E_{F}\right)($ States $/ \mathrm{eV})$ & & 3.446 & 3.350 & 3.302 & 3.116 & 2.802 \\
\hline
\end{tabular}

${ }^{a}$ from Ref. 13 . 


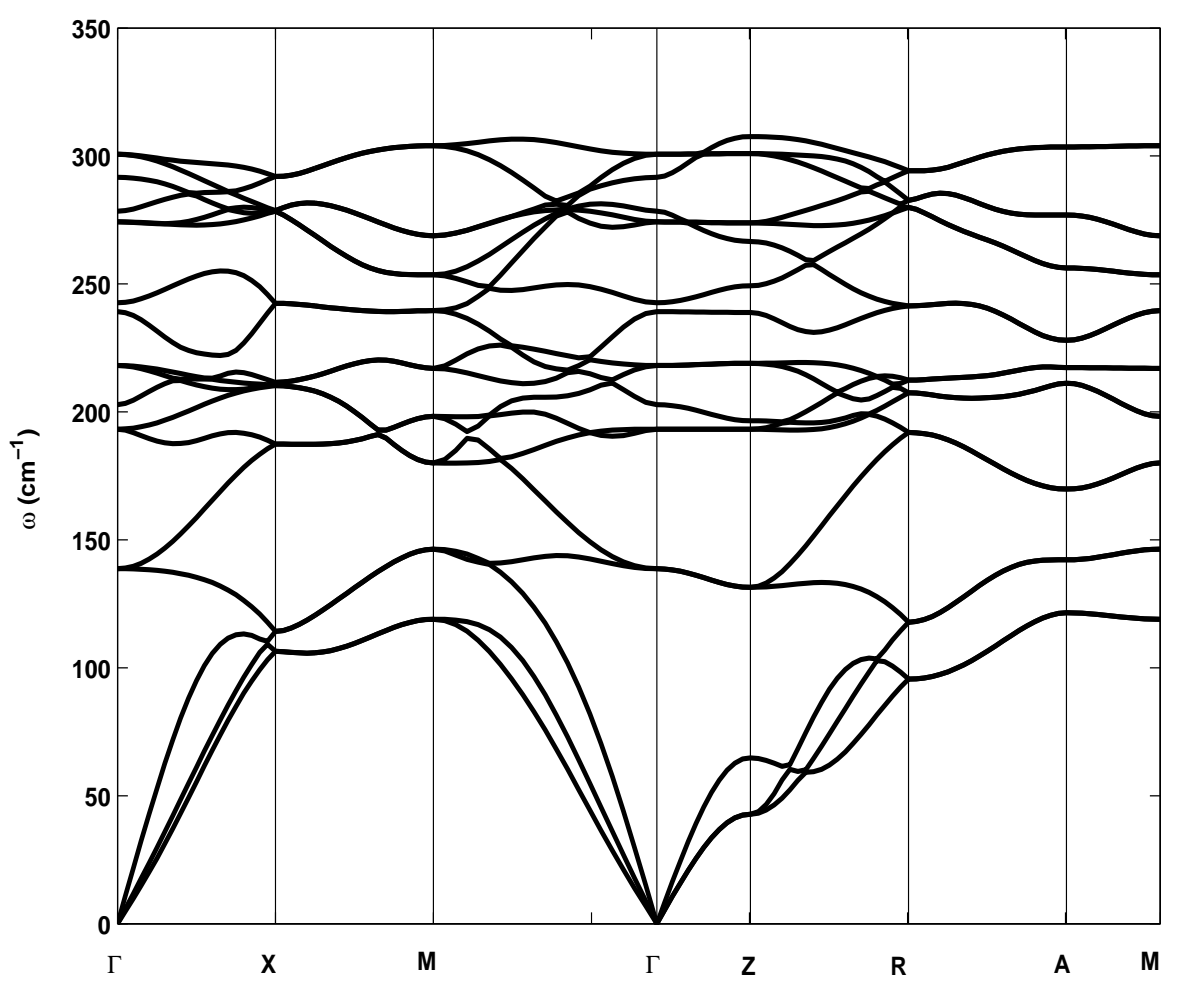

FIG. 1: The calculated phonon dispersion curves for LiFeAs 

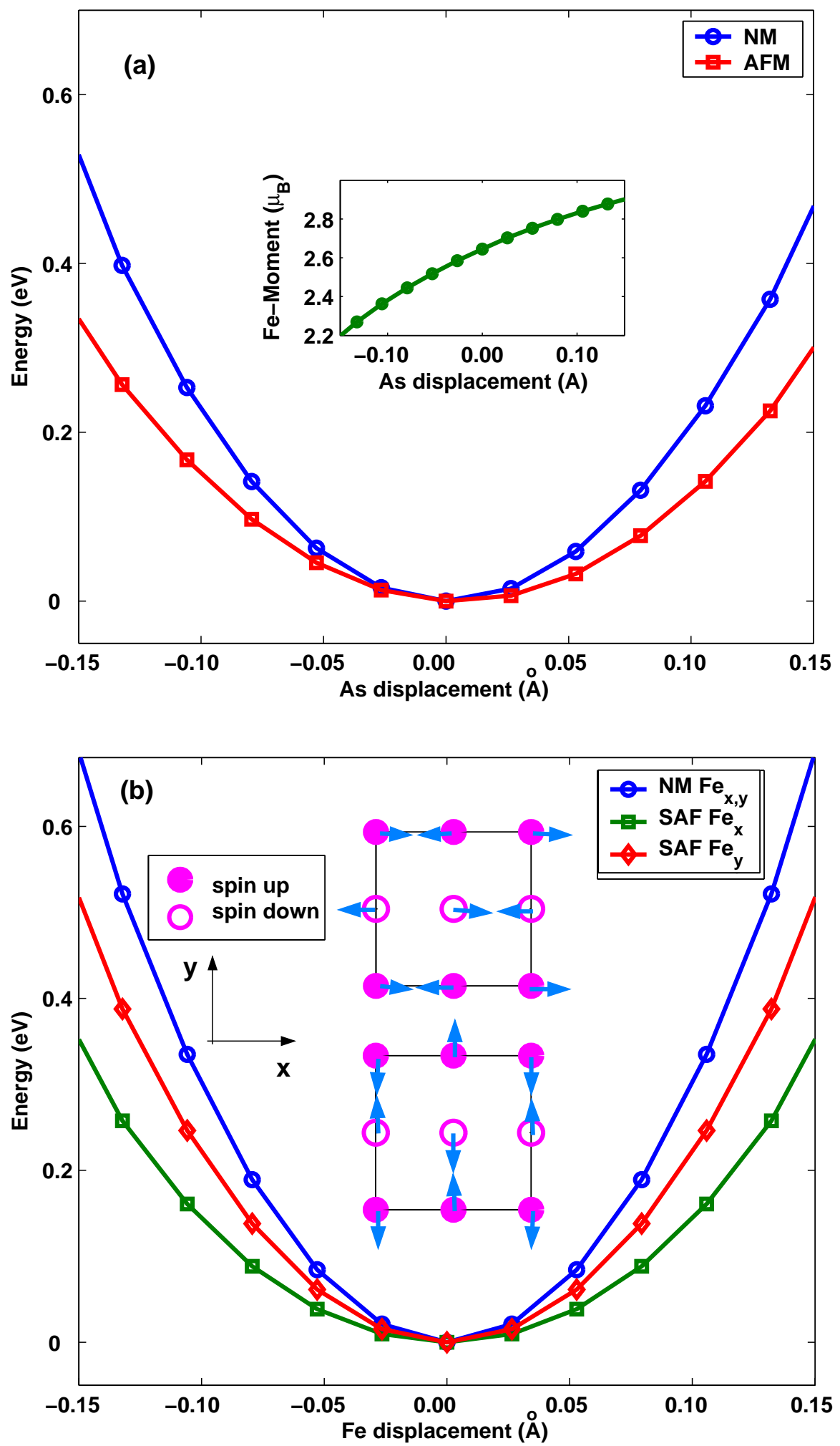

FIG. 2: Energy curves as a function of atom displacement. (a) for As $A_{g}$ mode and (b) for Fe $E_{g}$ mode. The calculated Fe-magnetic moment vs. As displacement is shown in the insert of (a), Negative As displacement corresponds to As atoms moving towards the Fe-plane. Phonon displacement patterns for the in-plane Fe $E_{g}$ mode are shown in the insert of (b). 